\title{
Removal of Organic Micropollutants from Water by Macrocycle- Containing Covalent Polymer Networks
}

\author{
Xiaofan Ji, ${ }^{*[a]}$ Hu Wang, ${ }^{[c]}$ Hongyu Wang, ${ }^{*[b]}$ Tian Zhao, ${ }^{[c]}$ Zachariah A. Page, ${ }^{*[c]}$ Niveen M. Khashab, ${ }^{[d]}$ \\ and Jonathan L. Sessler ${ }^{\star[c]}$
}

\begin{abstract}
Access to clean drinking water is a recognized societal need that touches on the health and livelihood of millions of people worldwide. This is providing an incentive to develop new watertreatment technologies. Traditional technologies, while widespread, are usually inefficient at removing organic pollutants from sewage or so-called grey water. Macrocycle-containing covalent polymer networks have begun to attract attention in the context of water treatment owing to the inherent stability provided by the polymer backbones and their ability to capture micropollutant guests as the result of tunable macrocycle-based host-guest interactions. In this minireview, we summarize recent advances (from 2016 to mid-2020) involving the removal of organic micropollutants from water using macrocycle-containing covalent polymer networks. An overview of future challenges within this subfield is also provided.
\end{abstract}

\section{Introduction}

Fresh water is one of the most important natural resources in the world. ${ }^{[1]}$ However, water pollution is threatening access to this resource, while adversely affecting aquatic life, human health, and our environment. Water issues also impact the energy, industrial, and agricultural sectors, and thus are directly tied to many facets of the economy.[2] In many instances, water contamination can be traced directly to human activity. ${ }^{[3]}$ For instance, various industrial endeavors can lead to the untoward release of wastewater containing hazardous compounds that often permeate into groundwater, lakes, rivers, estuaries and coastal seas. ${ }^{[3]}$ In addition, modern agriculture can serve as a

[a] Prof. X. Ji

School of Chemistry and Chemical Engineering

Key Laboratory of Material Chemistry for Energy Conversion

and Storage, Ministry of Education

Huazhong University of Science and Technology

Wuhan 430074, China

E-mail: xiaofanji@hust.edu.cn

[b] Prof. H. Wang, Prof. J. L. Sessler, T. Zhao

Department of Chemistry, College of Science, and Center for

Supramolecular Chemistry \& Catalysis

Shanghai University

Shangda Road, Shanghai, 200444, P. R. China

E-mail: wanghy@shu.edu.cn

[c] Dr. H. Wang, Prof. J. L. Sessler, Prof. Z. A. Page

Department of Chemistry

The University of Texas at Austin

105 E. 24th Street A5300, Austin, Texas 78712, United States

E-mail: zpage@cm.utexas.edu, sessler@cm.utexas.edu

[d] Prof. N. M. Khashab

Smart Hybrid Materials Laboratory, Physical Science and

Engineering Division, King Abdullah University of Science and

Technology (KAUST), 4700 King Abdullah University of Science and Technology, Thuwal 23955-6900, Kingdom of Saudi Arabia source of fertilizers and pesticides that then flow into various waterways. ${ }^{[3]}$ Other anthropogenic sources of pollution include oil, gasoline, biofuels, pharmaceutical waste, and pesticides. ${ }^{[3]}$ Increases in population are exacerbating the strain on freshwater resources. These combined stressors provide a compelling incentive to develop new technologies for removing pollutants from water.

Membrane filtration is a current water purification technology in widespread use. Often, however, membrane filtration is unable to lower the concentrations of contaminants to safe levels. ${ }^{[4]}$ Oxidative, electrochemical, photochemical, and sonochemical techniques have recently been developed to purify water. Unfortunately, such methods can generate degradation byproducts possessing some toxicity. ${ }^{[5]}$ As an alternative to degrading the micropollutants in water, various adsorbents have been explored, including activated carbon, carbon fibers, carbon nanotubes, and anion exchange resins. ${ }^{[4-6]}$ Currently, activated carbon (AC) is widely regarded as the leading adsorbent because of its low cost and decent adsorbance capacity. ${ }^{[7,8]}$ However, ACbased approaches are also subject to limitations, including slow uptake kinetics and low affinities for many relatively hydrophilic micropollutants..$^{[7,8]}$

Compared to traditional inorganic adsorbents, macrocyclecontaining covalent polymer networks, offer advantages in terms of removing organic micropollutants from water. The hybrid nature of these systems is considered key. First, the presence of covalent bonds endows the polymer network with high thermal and chemical stability while the use of relatively light organic constituents provides a gravimetric advantage. ${ }^{[6]}$ Second, the presence of macrocycles incorporated within the materials allows for the capture of various classes of guests. ${ }^{[9-13]}$ The inclusion of macrocycles is also expected to make regeneration of the polymer materials more facile. This is because macrocycle-based host-guest interactions are often sensitive to changes in the environmental conditions, including $\mathrm{pH}$, light, temperature, pressure, redox conditions, metal cations, anions, cations, solvent, and so on. ${ }^{[9-13]}$ Common macrocycles that have been used to create polymeric networks for water purification include cyclodextrins (CDs), ${ }^{[14-20]}$ calixarenes, ${ }^{[21-26]}$ pillararenes, ${ }^{[27-32]}$ resorcinarenes, ${ }^{[33-37]}$ and imidazolium macrocycles. ${ }^{[38-41]}$ On the other hand, other typical macrocycles, including crown ethers, ${ }^{[42-}$ ${ }^{44]}$ cucurbiturils $(\mathrm{CBs}),{ }^{[45-47]}$ and calix[4]pyrroles $(\mathrm{C} 4 \mathrm{Ps},)^{[48-50]}$ have not yet been widely used in this context. In this review, we highlight recent advances (from 2016 to mid-2020) in the removal of organic micropollutants from water by various macrocyclecontaining covalent polymer networks. 
WILEY-VCH

Xiaofan Ji completed his Ph.D. from Zhejiang University under the direction of Prof. Feihe Huang in June 2015. From December 2013 to May 2014, he visited the group of Prof. Steven C. Zimmerman at the University of Illinois at Urbana-Champaign. From September 2015 to January 2016, he was a JSPS Research Fellow in the University of Tokyo under the direction of Prof. Takuzo Aida. From June 2016 to September 2018, he was a postdoctoral fellow with Prof. Jonathan L. Sessler at the University of Texas at Austin. From September 2018 until December 2019, he was a postdoctoral fellow with Prof. Ben Zhong Tang at the Hong Kong University of Science and Technology. In December 2019 he joined the faculty of the Huazhong University of Science and Technology as a full professor. His research interests are 1) polymeric materials for water purification and 2) macroscopic assembly of fluorescent polymeric gels.

Hu Wang was born in Anhui, China in 1990. He received his bachelor of science degree in applied chemistry from Xidian University, China. In June 2019, He completed his Ph.D. from Zhejiang University under the direction of Prof. Feihe Huang. From November 2016 to May 2017, he visited the group of Prof. Rafal Klajn at Weizmann Institute of Science. From September 2019, he is a Postdoctoral Fellow jointly advised by Prof. Jonathan L. Sessler and Prof. Zak Page at The University of Texas at Austin. His current studies are focused on functional materials constructed by non-covalent interaction and traditional polymer.

Hongyu Wang received his $\mathrm{PhD}$ degree in macromolecular chemistry and physics from Fudan University in 2009. Then, he started to work at Shanghai University (SHU) and now is an Associate Professor at SHU. In 2017, he worked as a visiting scholar for one year at the lab of Professor Jonathan L. Sessler at the University of Texas at Austin. His research interests focus on synthesis of organic optoelectronic functional materials and their applications to organic electronic devices.
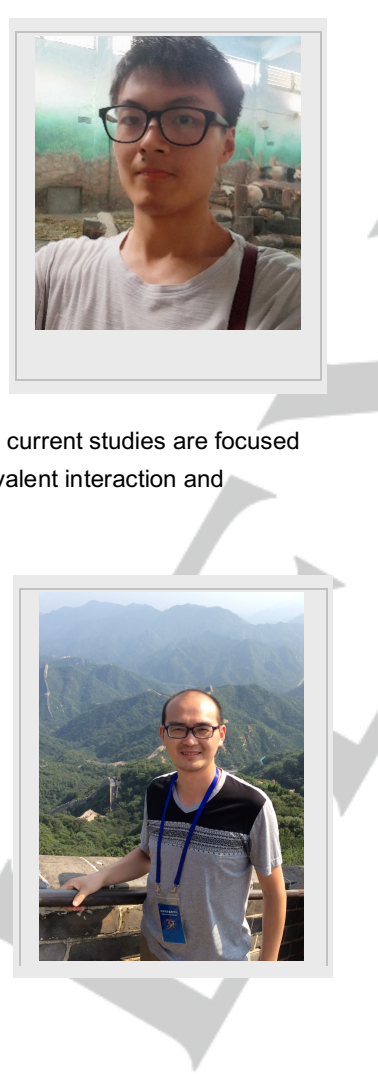

Tian Zhao was born in Gansu, China. He obtained his BS degree in chemistry at Tsinghua University (2014-2018). After graduation, he moved on to the University of Texas at Austin (2018-present). He is now pursuing his $\mathrm{PhD}$ degree in supramolecular chemistry under the supervision of Prof. Jonathan L. Sessler.

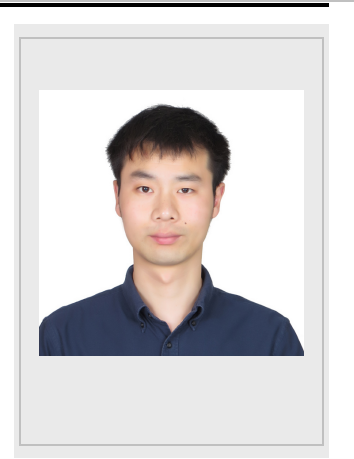

Zachariah A. Page received a $\mathrm{PhD}$ in Polymer Science and Engineering from the University of Massachusetts, Amherst in 2015. After completing postdoctoral research at the University of California, Santa Barbara in 2018, he began as an Assistant Professor of Chemistry at The University of Texas at Austin. His current research interests focus on the fabrication and manipulation of polymers with light.

Niveen M. Khashab is an Associate Professor in the Chemical Sciences Program at King Abdullah University of Science and Technology (KAUST). After her doctoral studies at the University of Florida, she joined Sir Fraser Stoddart's Lab at the University of California, Los Angeles, and then at Northwestern University. Her current research efforts are focused on intrinsically porous materials (IPMs) for energy intensive separations and the formation

supramolecular assembled capsules (SACs) for encapsulation and delivery.

Jonathan L. Sessler received a BSc degree in Chemistry in 1977 from the University of California, Berkeley and a PhD from Stanford University in 1982. After postdoctoral stays in Strasbourg and Kyoto, he accepted a position as an Assistant Professor of Chemistry at The University of Texas at Austin, where he is the DohertyWelch Chair. He has held a number of visiting professorships, including most recently (from September 2015-August 2020) at Shanghai University. His current hindex is 107 .

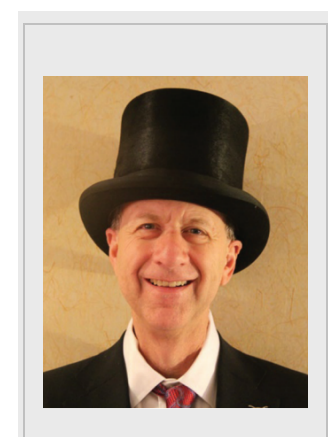

\section{Cyclodextrin-Containing Covalent Polymer Networks}

$\beta$-Cyclodextrin ( $\beta-C D)$ is a unique cyclic oligosaccharide composed of seven $\alpha-1,4$-linked D-glucopyranose units, with a cavity depth of ca. $7.9 \AA$ and a diameter of approximately $6 \AA .{ }^{[14]}$ The cavity of $\beta-C D$ is relatively hydrophobic and it forms hostguest inclusion complexes with a variety of guests via a combination of solvatophobic and van der Waals interactions. ${ }^{[15]}$ Because of its recognition features and a variety of other favorable physicochemical and biological properties, $\beta-C D$ s and their analogues have been used across of a wide range of supramolecular chemistry-related applications. ${ }^{[16]}$ Recently, $\beta$ CD-based polymers have also received considerable attention for their possible role in environmental remediation. ${ }^{[17-20]}$

In pioneering work reported in 2016, Dichtel and co-workers developed porous $\beta$-CD-containing polymers (P-CDPs) derived from the nucleophilic aromatic substitution of tetrafluoroterephthalonitrile (TFN) by the hydroxyl groups of $\beta-C D$. 
WILEY-VCH

\section{MINIREVIEW}

The resulting systems were found to be effective at adsorbing a variety of organic micropollutants (MPs) from water (Fig. 1). ${ }^{[17]}$ The Brunauer-Emmett-Teller surface areas ( $\left.S_{\mathrm{BET}}\right)$ of the P-CDPs could be tuned from $35 \sim 263 \mathrm{~m}^{2} \mathrm{~g}^{-1}$ by adjusting the molar feeding ratios of the TFN and $\beta-C D$ precursors during the polymerization. Bisphenol A (BPA), a component of plastics that has attracted concerns as an endocrine disruptor, was chosen as a first model pollutant. The uptake efficiency of a P-CDP was compared to that of a non-porous $\beta$-cyclodextrin (NP-CDP) and several activated carbons (ACs). Each adsorbent $\left(1 \mathrm{mg} \mathrm{mL}^{-1}\right)$ eventually removed most of the bisphenol A from a $0.1 \mathrm{mM}(22.8$ $\mathrm{mg} \mathrm{mL}^{-1}$ ) aqueous solution, corresponding to equilibrium uptake values of 19-24 mg bisphenol A per $\mathrm{g}$ adsorbent, with P-CDP (22 $\mathrm{mg} \mathrm{g}^{-1}$ ) being at the high end of this range. In the case of P-CDP $\sim 95 \%$ of the equilibrium uptake of BPA was achieved within $10 \mathrm{~s}$. In contrast, NP-CDP and a non-porous $\beta-C D$ polymer crosslinked with epichlorohydrin (EPI-CDP) required 0.5 and $1 \mathrm{~h}$ to reach equilibrium, respectively. Likewise, two types of mesoporous ACs, Brita AC $\left(S_{B E T}=507 \mathrm{~m}^{2} \mathrm{~g}^{-1}\right)$ and GAC $\left(S_{\mathrm{BET}}=612 \mathrm{~m}^{2} \mathrm{~g}^{-1}\right)$, were found to require more than 1 hour to reach equilibrium. A third $A C$, termed NAC $\left(S_{B E T}=984 \mathrm{~m}^{2} \mathrm{~g}^{-1}\right)$, required $10 \mathrm{~min}$ to reach equilibrium.

These researchers also evaluated the ability of P-CDP to remove pollutants of different size, functionality and hydrophobicity including simple aromatics, pharmaceuticals and several representative pesticides: 2,4-dichlorophenol, 1-naphthyl amine, 2-naphthol, bisphenol S, metolachlor, ethinyl oestradiol, and propranolol. These organic contaminants were found to be removed rapidly by P-CDP. For P-CDP the apparent pseudosecond-order rate constants (kobs) were 15 to 200 times greater than those recorded in the case of ACs and NP-CDP. An attractive feature of P-CDP is that it can be regenerated by rinsing with $\mathrm{MeOH}$ at room temperature, a recovery process that resulted in almost no decrease in performance.
Figure 1. (a) Chemical structure (left) and schematic structure (right) of P-CDP (b) Structures and key functions of various organic micropollutants.

Perfluorooctanoic acid (PFOA), which is environmentally persistent and difficult to degrade because of the high thermodynamic stability of the constituent C-F bonds, has emerged as an environmental and health crisis in many communities. In 2017 in an effort to target PFOA, the Dichtel group developed modified P-CDP systems containing perfluoroarene cross-linkers. In this case nucleophilic substitution of decafluorobiphenyl (DFB, 1) by $\beta-C D$, rather than the tetrafluoroterephthalonitrile (TFN, 2) used in the first generation systems, allowed access to the cross-linked polymer network DFB-CDP (Fig. 2) ${ }^{[18]}$ In contrast to the permanent porosity and high surface area of P-CDP, the DFB-CDP samples proved nonporous $\left(S_{\mathrm{BET}}<10 \mathrm{~m}^{2} \mathrm{~g}^{-1}\right.$ ). Nevertheless, DFB-CDP proved capable of removing PFOA to lower equilibrium concentrations than other adsorbents on an equivalent mass basis. For instance, a DFB-CDP polymer ([DFB-CDP] $=10 \mathrm{mg} \mathrm{L}^{-1}$ ) derived from a DFB: $\beta-C D$ feeding ratio of $3: 1$ reduced initial $P F O A$ concentrations of $[\mathrm{PFOA}]=1 \mu \mathrm{g} \mathrm{L}^{-1}$ in water to $<10 \mathrm{ng} \mathrm{L}^{-1}$ within $24 \mathrm{~h}$. This final concentration is at least 7 times lower than the 2016 level set by the U.S. Environmental Protection Agency (70 ng $\left.\mathrm{L}^{-1}\right)$. In contrast to DFB-CDP, a high-end sieved coconut shell activated carbon (CCAC) and P-CDP removed only $56.3 \%$ and around $0 \%$ of the PFOA, respectively. Additionally, DFB-CDP could be regenerated and reused multiple times with no apparent decrease in performance by simply washing with $\mathrm{MeOH}$ at room temperature.
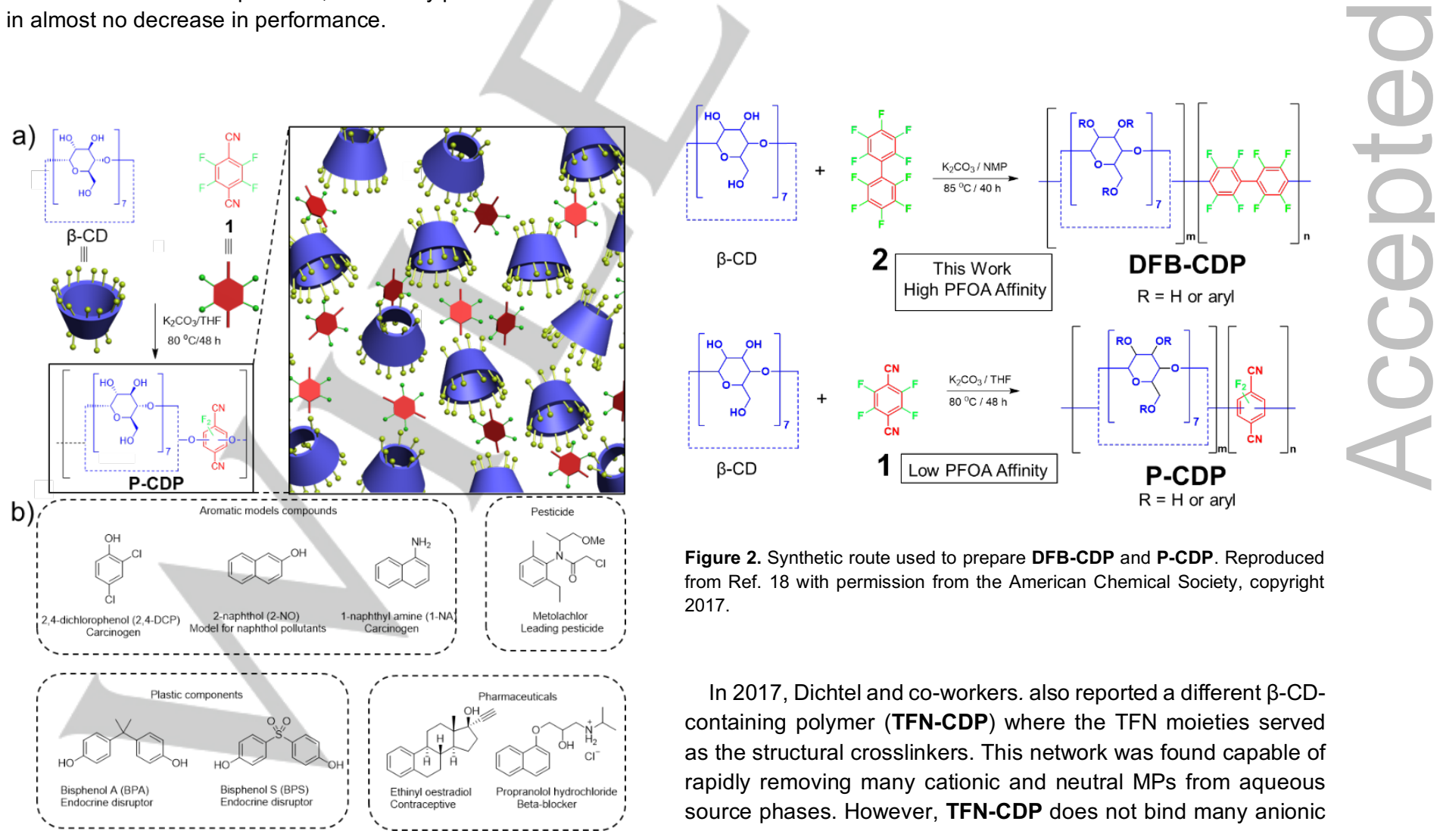

Figure 2. Synthetic route used to prepare DFB-CDP and P-CDP. Reproduced from Ref. 18 with permission from the American Chemical Society, copyright 2017.

In 2017, Dichtel and co-workers. also reported a different $\beta$-CDcontaining polymer (TFN-CDP) where the TFN moieties served as the structural crosslinkers. This network was found capable of rapidly removing many cationic and neutral MPs from aqueous source phases. However, TFN-CDP does not bind many anionic 
WILEY-VCH

\section{MINIREVIEW}

MPs, including anionic per- and polyfluorinated alkyl substances (PFAS), strongly. To address this limitation, Dichtel and coworkers reduced the nitrile groups in TFN-CDP to the corresponding primary amines. This served to reverse the affinity towards anionic MPs (Fig. 3), presumably as the result of amine protonation. ${ }^{[19]}$ The adsorption coefficients (log $K_{\mathrm{D}}$ values) of 91 structurally diverse MPs $\left([M P]_{0}=2 \mathrm{mg} \mathrm{L}^{-1}\right)$ for TFN-CDP and the reduced TFN-CDP (25 mg L-1) were measured. TFN-CDP exhibited $\log K_{D}$ of 2 3 for cationic MPs and $-0.5 \sim 1.5$ for anionic MPs, whereas the reduced TFN-CDP exhibited log $K_{D}$ values of 0.5 1.5 for cationic MPs and 2 4 for anionic MPs with anionic PFAS proving to be particularly effective substrates.

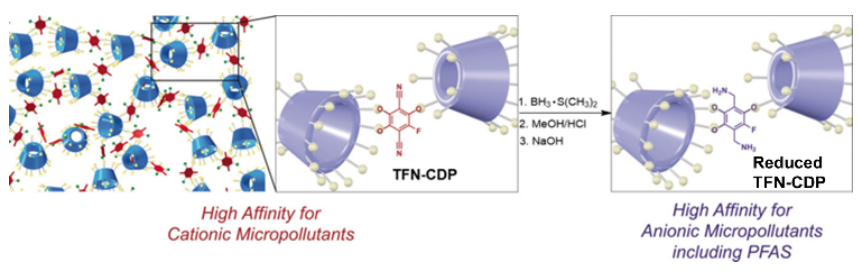

Figure 3. Post-polymerization reduction of TFN-CDP yields the amine functionalized reduced form of TFN-CDP. Reproduced from Ref. 19 with permission from Wiley-VCH, copyright 2019.

Recently, Wang and co-workers. reported a series of $\beta-C D-$ based crosslinked polymeric adsorbents (Fig. 4), PCD-VI, synthesized via the radical copolymerization of methacrylicmodified $\beta-C D(M C D)$ and 1-vinylimidazole (VI) using different monomer feeding ratios. ${ }^{[20]}$ Polymer PCD-VI exhibited excellent adsorption capacities for cationic rhodamine B (RB), anionic Congo Red (CR), and cadmium ion (Cd(II)) when tested separately as model contaminants in water. Importantly, PCD-VI was not plagued by "competitive adsorption" limitations when it was exposed to an aqueous mixture of RB and $\mathrm{Cd}(\mathrm{II})$. In fact, synergy was seen between RB and Cd(II). Both pollutants could then be recovered independently.
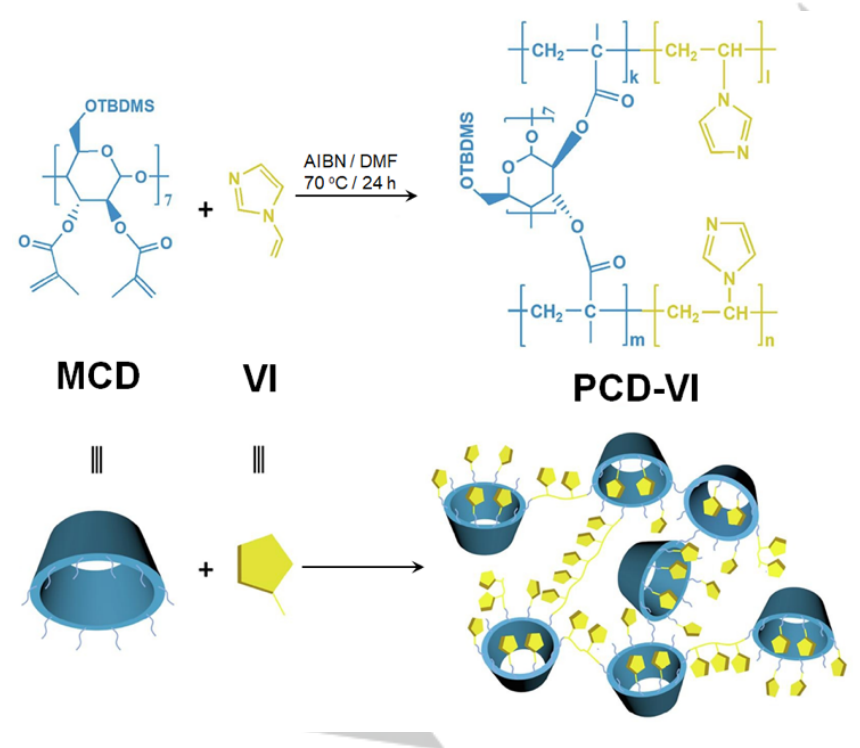

Figure 4. Synthetic strategy used to access PCD-VI (top) and schematic representation of PCD-VI and its precursors. Reproduced from Ref. 20 with permission from Elsevier, copyright 2019.

\section{Calixarene-Containing Covalent Polymer Networks}

Calixarenes, with their unique three-dimensional shapes and conformationally rigid structures, are one of the best known classes of macrocyclic hosts. ${ }^{[21]}$ They have been broadly exploited in all areas of supramolecular chemistry over the past three decades, including in sensing, catalysis, extraction, molecular encapsulation, and as the basis for rotaxanes and molecular machines. ${ }^{[22,23]}$ Calixarene-based materials have also been employed in gas separation and to target organic MPs.

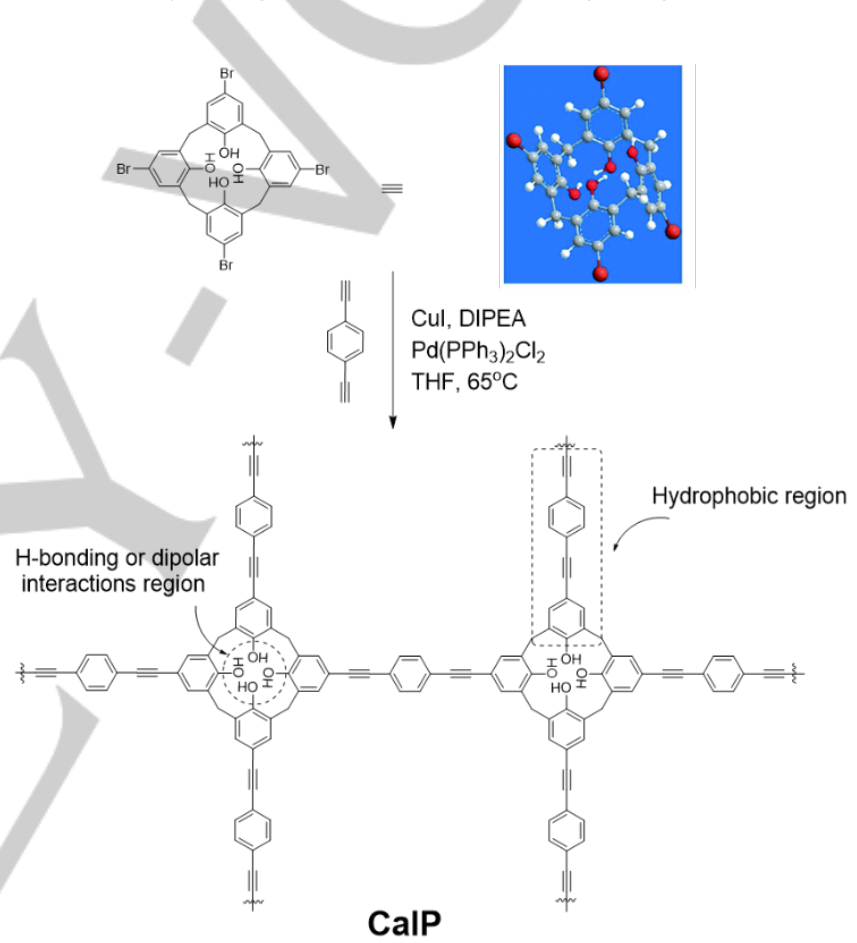

Figure 5. Top: Schematic illustration of CalP and synthetic route used to access this class of porous calix[4]arene-based polymer. Plausible sites on the polymer for interaction with pollutants are marked by dotted lines. Reproduced from Ref. 24 with permission from The Royal Society of Chemistry, copyright 2017.

In 2017, Trabolsi and co-workers reported the first porous covalent calix[4]arene-based polymer (CaIP) for water purification. CalP was synthesized via the palladium catalyzed SonogashiraHagihara cross-coupling of tetrabromo-calix[4]arene-tetrol and 1,4-diethynylbenzene monomers (Fig. 5). ${ }^{[24]}$ Its constituent aromatic rings and alkyne-rich backbone endowed CalP with superhydrophobic character with an average contact angle of $155.4 \pm 4.01$ degrees. The pores within CalP were found to be mainly located in the mesopore region, with a Barrett-JoynerHalenda (BJH) average pore diameter of $95 \AA$ and a high SBET of $596 \mathrm{~m}^{2} \mathrm{~g}^{-1}$. CalP was shown to quickly adsorb crude oil, engine oil, and organic solvents (toluene, EtOH, tetraethylene glycol) from water. In addition, the anionic dye, $\mathrm{CR}$, and two cationic dyes, methylene blue (MB) and rhodamine $B(R h B)$, could be effectively removed from water. About $80 \%$ of the $C R$ dye $(0.5 \mathrm{mM})$ was 
WILEY-VCH

\section{MINIREVIEW}

adsorbed by CalP $\left(0.5 \mathrm{mg} \mathrm{mL}^{-1}\right)$ in the first 5 minutes and essentially complete removal was observed within 15 minutes, giving a maximum adsorption capacity $Q_{m}$ of $673 \mathrm{mg} \mathrm{g}^{-1}$. For MB and $\mathrm{RhB}$, about $95 \%$ and $50 \%$ were removed within 5 minutes, respectively, with the maximum adsorption capacities for $\mathrm{MB}$ and RhB being 625 and $484 \mathrm{mg} \mathrm{g}^{-1}$.

a)

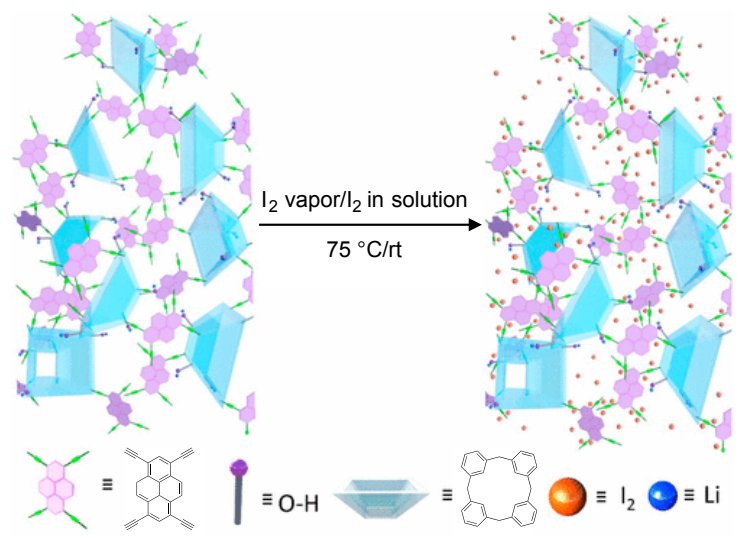

b)

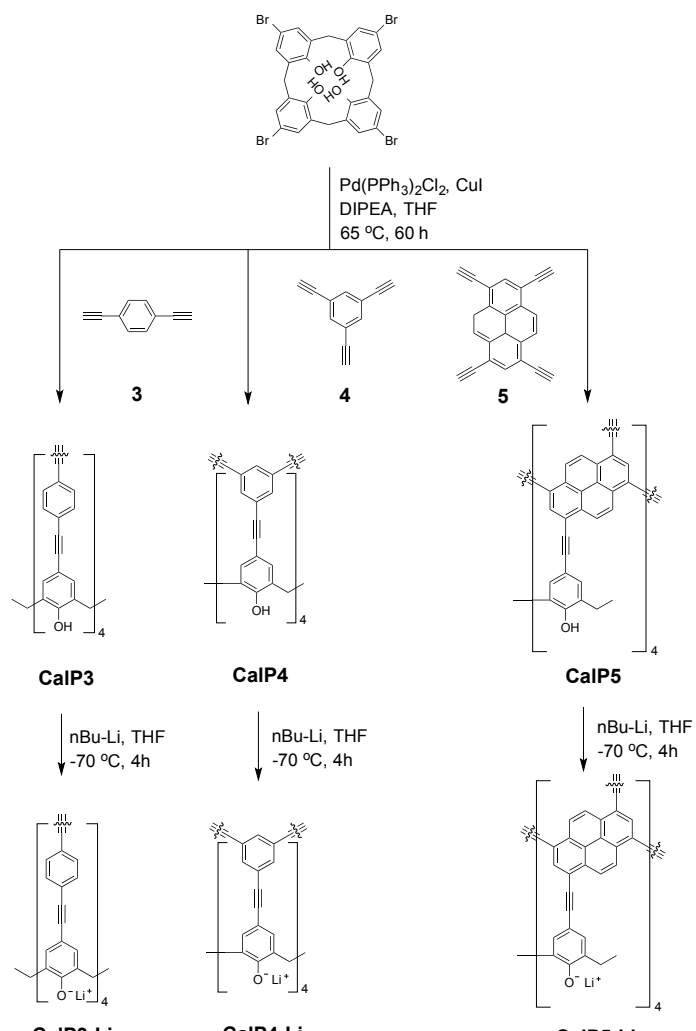

CalP3-Li

CalP4-Li

m.n polymeric extension

Figure 6. Top: Schematic representation of $\mathrm{I}_{2}$ adsorption by CalPns. Bottom Synthetic route used to access porous calix[4]arene polymers (CaIPns) via Sonogashira-Hagihara cross-coupling. Follow-up lithiation is also shown. Reproduced from Ref. 25 with permission from the American Chemical Society, copyright 2017

Radioactive iodine $\left(I_{2}\right.$ containing ${ }^{129} \mid$ and/or $\left.{ }^{131} I\right)$ is a volatile solid that, when accidently released as a gas in nuclear power plant explosions, poses a serious threat to the public and environment. To capture $\mathrm{I}_{2}$, Trabolsi and co-workers prepared a series of hyper-cross-linked $\pi$-bond-rich porous covalent polycalix[4]arenes (CaIPns, $n=3-5$, CalP3, CalP4, and CalP5) via Sonogashira-Hagihara cross-coupling of tetrabromocalix[4]arene-tetrol with 1,4-diethynylbenzene (3), 1,3,5triethynylbenzene (4), and 1,3,6,8-tetraethynylpyrene (5) monomers, respectively (Fig. 6). ${ }^{[25]}$ The corresponding lithiated polymers: CalP3-Li, CalP4-Li, and CalP5-Li, were also synthesized with the goal of enhancing the adsorption properties of the materials by providing sites for charge induced dipolar and quadrupolar electrostatic interactions. BET model studies yielded $S_{\text {BET }}$ values of 596,630 , and $759 \mathrm{~m}^{2} \mathrm{~g}^{-1}$ for CalP3, CalP4, and CalP5, respectively. After lithiation, the $S_{B E T}$ values were lower (i.e., 274, 308, and $445 \mathrm{~m}^{2} \mathrm{~g}^{-1}$ for CalP3-Li, CalP4-Li, and CalP5$\mathrm{Li}$, respectively). All polymers proved capable of adsorbing $\mathrm{I}_{2}$ from solution and as vapors. The lithiated polymers generally proved faster and more efficient at this uptake than their non-lithiated counterparts. Among all polymers subject to study, CalP5-Li proved especially fast and efficient, requiring only 30 minutes to achieve near-complete adsorption.

Subsequently, the Trabolsi group found that the CalPns could adsorb organic MPs from water. ${ }^{[26]}$ The absorption profiles for four pollutants, BPA, 1-naphthylamine (1-NA), 2-naphthol (2-NO), and propranolol, were determined. Of the three CalPns tested, CalP5 exhibited the fastest adsorption kinetics and the highest adsorption capacity. For example, CalP5 removed $\sim 70 \%$ of BPA from an aqueous source phase within $30 \mathrm{~s}$ and achieved an equilibrium removal of $\sim 80 \%$ after $15 \mathrm{~min}$. The $k_{\text {obs }}$ for the uptake of BPA by CalP5 was $2.12 \mathrm{mg} \mathrm{g}^{-1} \mathrm{~min}^{-1}$, which is significantly higher (by 16-240 times) than that of ACs and 1.4 times higher than P-CDP (the most efficient material previously reported). Moreover, these polymers could be regenerated by washing with ethanol at room temperature and were found to maintain their adsorption efficiencies over multiple cycles.

\section{Pillar[n]arene-Containing Covalent Polymer Networks}

Pillararenes, a relatively new class of supramolecular hosts, have attracted considerable attention since their discovery by Ogoshi and co-workers in 2008. ${ }^{[27]}$ In contrast to meta-bridged calixarenes with basket-shaped structures, pillararenes are linked by methylene bridges at the para positions of the constituent 2,5dialkoxybenzene derivatives. ${ }^{[28]}$ Pillar[n]arenes $(P n, n=5-10)$, especially P5 and P6, display remarkable host-guest recognition properties and have been explored in a range of applications. ${ }^{[29]}$ Recently, pillararene-based porous polymer networks have been reported in the context of water purification. For instance, Huang and co-workers reported a carboxyl-derived pillar[5]arene-based 3D network polymer (Fig. 7), P-(P5-PPD), and studied its ability to remove organic MPs from water. ${ }^{[30]}$ These researchers evaluated a series of natural and anthropogenic contaminants considered harmful to both humans and the environment: BPA, metolachlor, amoxicillin sodium, fluorescein sodium, methyl orange, methyl blue, 2,4-dichlorophenol (2,4-DCP), 2-naphthol (2-NO), and 1-naphthyl amine (1-NA). It was found that P-(P5PPD) could effectively remove the organic MPs from water, displaying pollutant removal efficiencies that exceeded those of the ACs. In fact, P-(P5-PPD) was found to remove fluorescein 
WILEY-VCH

\section{MINIREVIEW}

sodium (96.4\%) and methyl orange (95.2\%) almost completely from water with equilibrium uptakes reaching $360.8 \mathrm{mg} \mathrm{g}^{-1}$ and $311.6 \mathrm{mg} \mathrm{g}^{-1}$, respectively. Fast adsorption kinetics were also seen, with the maximum adsorption levels typically being reached in under $1.0 \mathrm{~min}$. Moreover, P-(P5-PPD) could be regenerated by simply washing with DMF and acetone at room temperature.

a)

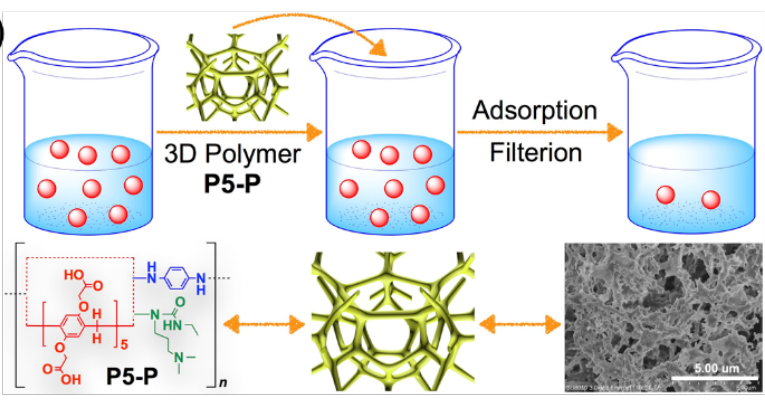

b)

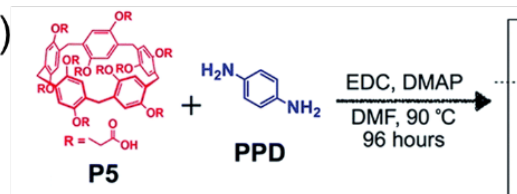

c)
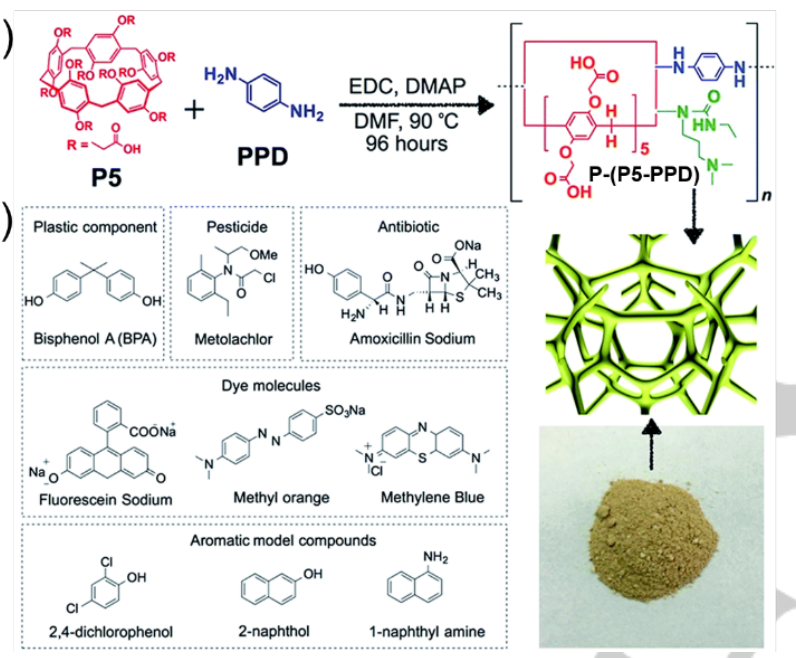

Figure 7. (a) Schematic illustration of the adsorption process proposed in the case of P5-P. (b) Synthetic route to P5-P. (c) Structures of the organic MPs tested as substrates for P5-P. Reproduced from Ref. 30 with permission from The Royal Society of Chemistry, copyright 2017.

In 2017, Ma and co-workers reported two pillar[n]arenes-based porous polymers, P-(P5-TFTN) and P-(P6-TFTN). These systems were obtained by using tetrafluoroterephthalonitrile (TFTN) to cross-link the phenolic hydroxyl groups of P5 and P6 (Fig. 8), ${ }^{[11]}$ respectively. Both pillar[n]arenes-based porous polymers were able to remove various MPs from water and do so rapidly and efficiently. Model MPs tested included an herbicide (paraquat), a metal cation $\left(\mathrm{Cu}^{2+}\right)$ a pharmaceutical compound (carbamazepine), an antibiotic (tetracycline), a plastic component (BPA), and simple aromatics (1-naphthyl amine, 2,4dichlorophenol, and 2-naphthol). The adsorption proved to be especially efficient in the case of paraquat for which very fast uptake kinetics $\left(k_{\mathrm{obs}}=33.3 \mathrm{~g} \mathrm{mg}^{-1} \mathrm{~min}^{-1}\right)$ were seen. The maximum adsorption capacity $q_{\max }$ of $\mathbf{P}$-(P5-TFTN) was calculated to be $209 \mathrm{mg} \mathrm{g}^{-1}$.

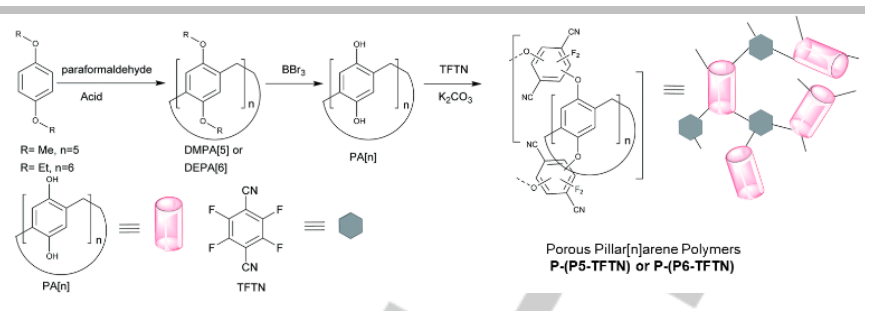

Figure 8. Synthesis of porous pillar[n]arene polymers. Reproduced from Ref. 31 with permission from The Royal Society of Chemistry, copyright 2017.

Liu and co-workers reported two macrocycle-containing mesoporous polymer membranes, P-(SC4-TC) and P-(P5-TC), prepared via the interfacial polymerization of terephthaloyl chloride (TC) with P5 and sulfonatocalix[4]arene (SC4), respectively (Fig. 9). ${ }^{[32]}$ The $\mathrm{pH}$ value of the aqueous phase was vital for the fabrication of the films. For fabricating the P-(SC4-TC) films, $5 \%$ SC4 with a $1 \% \mathrm{NaOH}$ aqueous phase and $0.5 \%$ TC in n-hexane proved to be the optimal reaction conditions. While $2 \%$ $\mathrm{P} 5$ and a $1.6 \% \mathrm{NaOH}$ aqueous phase with $0.5 \% \mathrm{TC}$ in $\mathrm{n}$-hexane proved effective for the preparation of P-(P5-TC) films. The $S_{\mathrm{BET}}$ values for the P-(SC4-TC) and P-(P5-TC) films were 123 and 85 $\mathrm{m}^{2} \mathrm{~g}^{-1}$, respectively. Both polymeric films proved effective at removing cationic dyes, such as brilliant green, basic red, and thiazole orange from aqueous media. Furthermore, these polymers could be used to separate cationic dyes (e.g., malachite green oxalate) from anionic dyes (e.g., sulforhodamine B). Gratifyingly, the absorbed dyes could be easily eluted from the polymers using an ethanol wash, thereby allowing the films to be restored to their original states.

a)
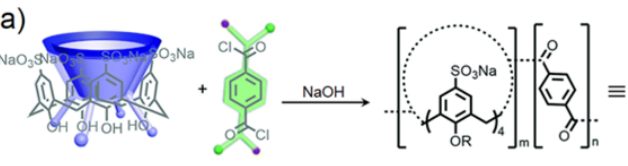
P-(SC4-TC)
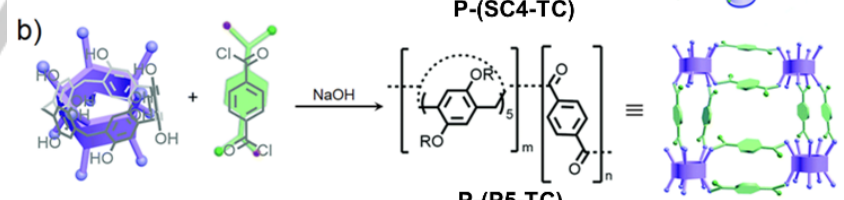

Figure 9. P-(SC4-TC) and P-(P5-TC) made via interfacial polymerization. (a and b) Synthetic routes to films starting from $\mathbf{S C} 4$ or $\mathbf{P 5}$ (dissolved in $\mathrm{NaOH}$ solution) and TC (dissolved in organic phase), respectively. Reproduced from Ref. 32 with permission from The Royal Society of Chemistry, copyright 2018.

\section{Resorcinarene-Containing Covalent Polymer Networks}

Calix[4]resorcinarenes, typically bowl-shaped molecules with eight phenolic groups in their upper rims, ${ }^{[33]}$ are recognized as being excellent supramolecular hosts capable of encapsulating a variety of guests. ${ }^{[34,35]}$

With the goal of capturing radioactive $I_{2}$ from nuclear waste streams, Yan and co-workers designed a series of azo-bridged calix[4]resorcinarene-based porous organic frameworks (CalPOFs). These systems were prepared via the diazo coupling of 4,4'-biphenyldiamine with various C-alkylcalix[4]resorcinarenes 
WILEY-VCH

\section{MINIREVIEW}

( $\mathrm{RsC}_{\mathrm{n}} \mathrm{s} ; \mathrm{n}$ stands for the length of the chain in the meso-alkyl substituent) under mild conditions (Fig. 10). ${ }^{[36]}$ The resulting CalPOFs display permanent porosity with $S_{\text {BET }}$ values of 303,154 and $91 \mathrm{~m}^{2} \mathrm{~g}^{-1}$ being recorded for CalPOF-1, CalPOF-2 and CalPOF-3, respectively. These materials were found to take up $I_{2}$ vapor effectively as follows: CalPOF-1 (477 wt \%) > CaIPOF-2 (406 wt \%) > CalPOF-3 (353 wt \%).

a)
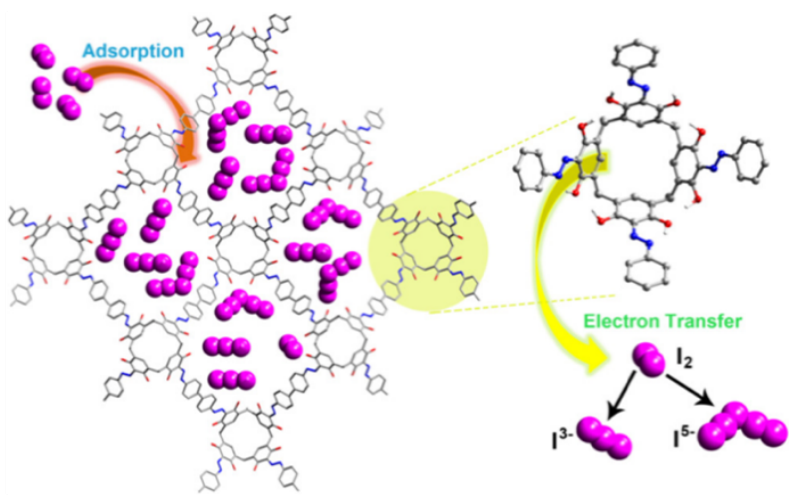

b)
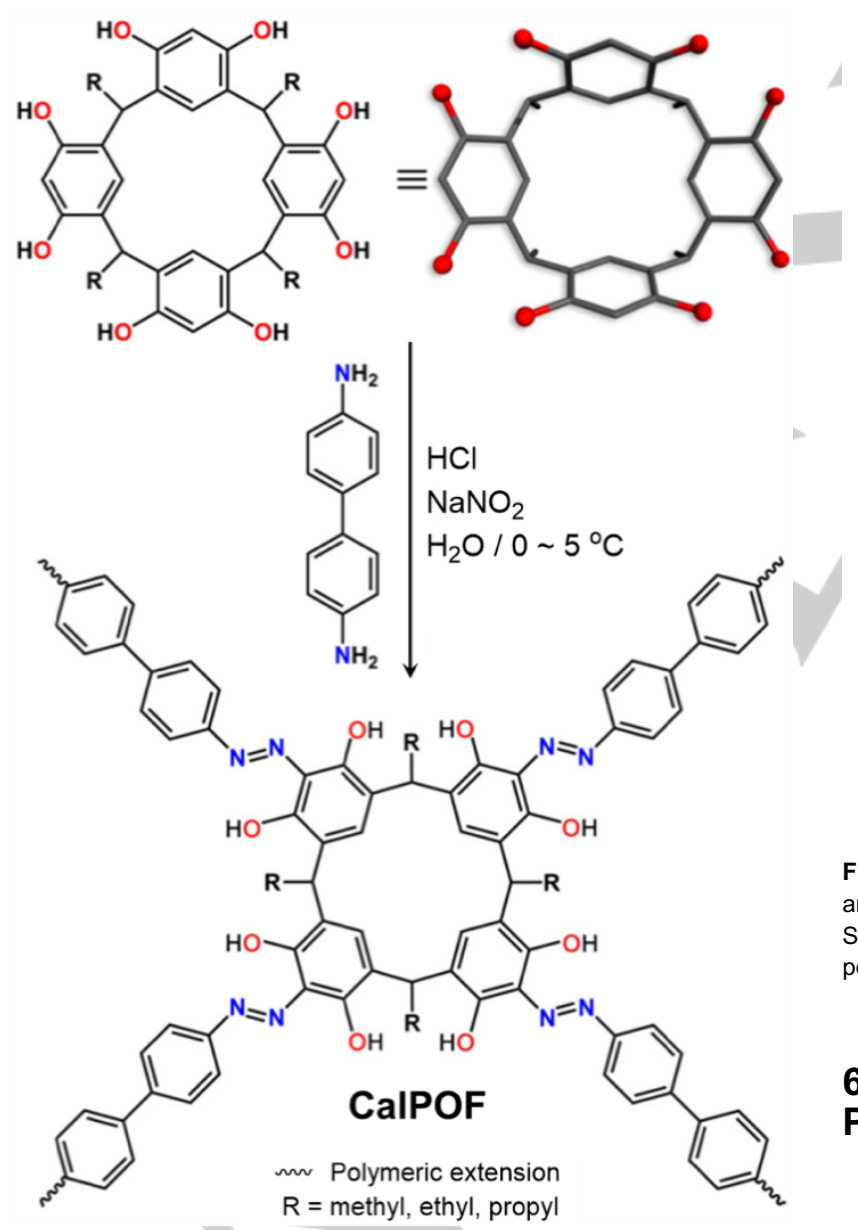

Figure 10. Top: Schematic representation of $\mathrm{I}_{2}$ adsorption by CalPOF. Bottom: Synthesis route leading to CalPOF involving the diazo coupling between diazonium salts and RsCns. Reproduced from Ref. 36 with permission from the American Chemical Society, copyright 2018.
Halomethanes $\left(\mathrm{CHCl}_{3}, \mathrm{CH}_{2} \mathrm{Cl}_{2}\right.$, etc. $)$ are among the most common organic micropollutants present in drinking water. Currently, the most effective approach to removing $\mathrm{CHCl}_{3}$ from drinking water is adsorption by activated carbon substrates. However, ACs have relatively low affinity for trihalomethanes, especially $\mathrm{CHCl}_{3}$. As a result, many municipalities provide drinking water near or above the US EPA limit of $80 \mu \mathrm{g} \mathrm{L}^{-1}$ total trihalomethane content. Recently, Dichtel et al. integrated resorcinarene into porous polymer networks and showed that the resulting materials were able to remove trihalomethane from water (Fig. 11). ${ }^{[3]}$ The preparation of these materials relied on nucleophilic aromatic substitution of tetrafluoroisonicotinonitrile (TFIN) or TFN (2) with resorcinarene to give the cavitandcontaining polymers CP-TFIN and CP-TFN, respectively. The polymers prepared using the TFIN linker consistently demonstrated a higher removal percentage for all the MPs tested. For instance, CP-TFIN was found to remove over $85 \%$ of the $\mathrm{CHCl}_{3}$ from an aqueous source phase leaving a residual $\mathrm{CHCl}_{3}$ concentration below $15 \mu \mathrm{g} \mathrm{L}-1$.

a)

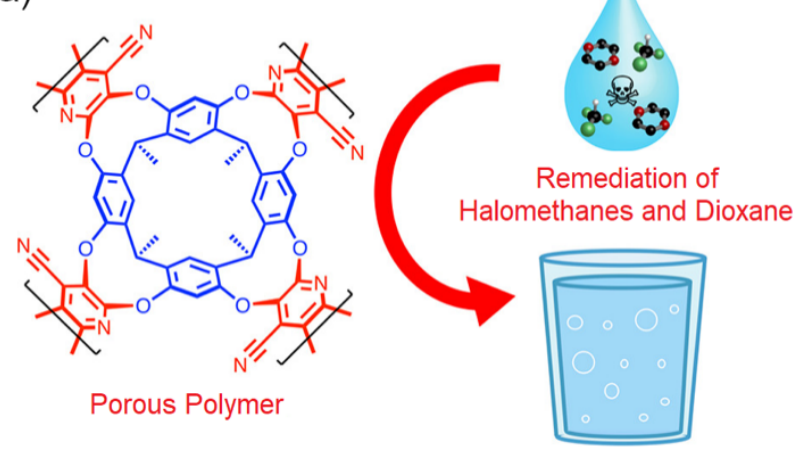

b)
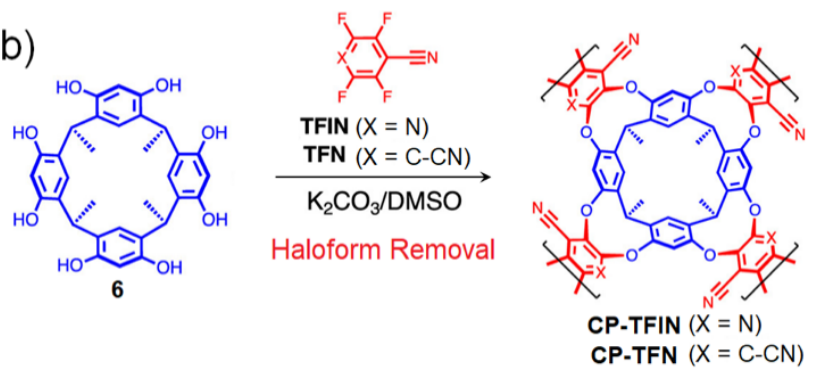

Figure 11. Top: Schematic illustration showing the removal of halomethanes and dioxane by porous cavitand polymers, CP-TFIN and CP-TFN. Bottom: Synthesis of porous cavitand polymers. Reproduced from Ref. 37 with permission from the American Chemical Society, copyright 2019.

\section{6. "Texas-Sized" Box-Containing Covalent Polymer Networks}

In 2010, Sessler and co-workers reported a flexible tetracationic imidazolium-based macrocycle, a 'Texas-sized' molecular box that in the presence of certain mono- and biscarboxylate anions would self-assemble into pseudorotaxanes or poly-pseudorotaxanes. ${ }^{[38]}$ The flexible and electron-deficient nature of this molecular box allows it to accommodate electronrich anionic guest molecules of different shapes and sizes. ${ }^{[39]}$ The resulting pseudorotaxane structures proved responsive to 
WILEY-VCH

\section{MINIREVIEW}

changes in $\mathrm{pH}$ and temperature; in particular, self-assembly could be effectively "turned off" by lowering the $\mathrm{pH}^{.}{ }^{[40]}$

One example of a polymeric network based on the "Texassized" box was reported by Sessler and co-workers (Fig. 12). In this study the tetracationic box macrocycle was used to crosslink poly(vinyl alcohol) (PVA) to give a hydrophilic polymer network, P-TxSB. ${ }^{[41]}$ This network was found capable of capturing a variety of different inorganic anions (viz. $\mathrm{NO}_{3}^{-}, \mathrm{NO}_{2}{ }^{-}, \mathrm{SO}_{4}{ }^{2-}, \mathrm{HS}^{-}, \mathrm{HSO}_{4}^{-}$, and $\mathrm{F}^{-}$) and organic anions (e.g., fluorescein, flavin mononucleotide, Lucifer yellow, and resorufin) from water. This capture resulted in the formation of anion salt-containing hydrogels. Removal of the anions could then be achieved by lifting the resulting hydrogels out of the aqueous phase. Treating the anion-containing hydrogels with dilute $\mathrm{HCl}$ was then found to trigger the protonation-induced release of the bound anions, allowing the hydrogels to be recycled for reuse. This macrocyclecontaining hydrogel provides a potentially attractive approach for removing by physical means undesired anions from aqueous environments.

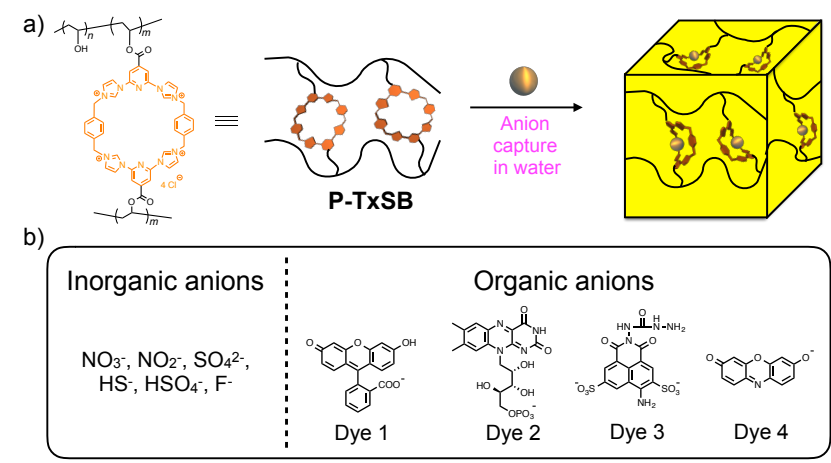

c)

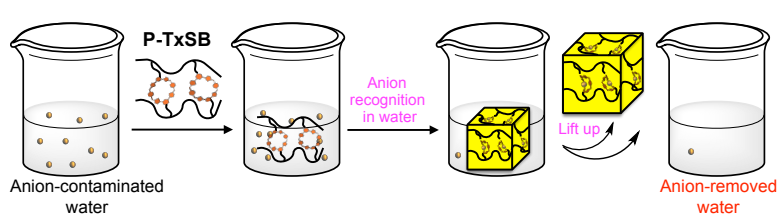

Figure 12. (a) Chemical structures and cartoon representations of polymeric materials based on the 'Texas-sized' molecular box. (b) Proposed macrocycle-anion binding interactions that are expected to be operative in aqueous environments. (c) Anion removal from water by use of a polymeric network P-TxSB that contains the 'Texas-sized' molecular box, which functions as a tetracationic macrocyclic anion receptor. Reproduced from Ref. 41 with permission from the American Chemical Society, copyright 2019.

\section{Summary and Outlook}

In this review, the use of macrocycle-containing covalent polymer networks for the removal of organic micropollutants from water has been summarized. To date, a number of macrocycles, including CDs, calixarenes, pillararenes, resorcinarenes, and imidazolium macrocycles, have been incorporated into covalent polymer networks targeted for use in water purification. Impressive results have been obtained in terms of capturing specific pollutants. However, there remains considerable room for further advances. For example, within the CD family, effort to date has mainly focused on $\beta-C D$; in contrast, $\alpha-C D$ and $\gamma-C D$ have been rarely used to create polymer networks. The development of $\alpha$-CD- and $\gamma$-CD-based polymers could capture smaller and larger pollutants, respectively, from water. Similarly, emphasis in terms of materials-related work has been placed on calix[4]arenebased systems, while larger calix[n]arenes $(n=5,6,8)$ remain underexplored. Likewise larger pillar[n]arenes, such as pillar[8]arenes, pillar[9]arenes, and pillar[10]arenes, remain to be explored in the context of porous materials development. In the area of charged receptors, only a single first generation tetracationic imidazolium macrocycle has been used to create porous polymeric materials. Many other systems with different sizes or structures could prove useful in the context of porous material construction. In addition, other classic receptor systems, including crown ethers, cucurbiturils (CBs), or calix[4]pyrroles (C4Ps) have not yet been widely used to create porous networks targeting water purification. By expanding the scope of the receptor chemistry and adjusting further the polymer structure, we predict that new and more effective polymer networks can be produced that will allow the specific and targeted removal of organic micropollutants from water. Given the urgency of the problem, we expect to see further focused efforts in this area in the months and years ahead. In this context, macrocycle containing POPs are expected to offer an advantage. Since various supramolecular macrocycles can selectively bind small molecules, they are expected to be able to capture and remove targeted organic micropollutants from water with greater precision and fidelity than non-macrocycle containing POPs. The wide pallete of available macrocycles should help see this vision realized even more effectively than has been demonstrated to date. We thus predict that further dedicated research involving macrocycle containing POPs will lead to advances in supramolecular chemistry and materials science that will benefit directly both human health and the environment.

\section{Acknowledgements}

X. Ji. acknowledges initial funding from the Huazhong University of Science and Technology, where he is being supported by Fundamental Research Funds for the Central Universities (grant 2020kfyXJJS013). H.W. is grateful for supports from the National Natural Science Foundation of China (21975153) and Shanghai Pujiang Program (2019PJD017) for financial support. Acknowledgment is also made to the University of Texas System for a CONACYT Collaborative Research Grant to J.L.S. and the Robert A. Welch Foundation (grants F-0018 to J.L.S. and F-2007 to Z.A.P.) and the King Abdullah University of Science and Technology (OSR-2019-CRG-4032 to J.L.S. and N.M.K.)

Keywords: host-guest interactions $\cdot$ macrocycle $\cdot$ polymer networks $\cdot$ self-assembly $\cdot$ water-purification

[1] C. J. Vörösmarty, P. B. Mclntyre, M. O. Gessner, D. Dudgeon, A. Prusevich, P. Green, S. Glidden, S. E. Bunn, C. A. Sullivan, C. R. Liermann, P. M. Davies, Nature 2010, 467, 555-561.

[2] R. P. Schwarzenbach, B. I. Escher, K. Fenner, T. B. Hofstetter, C. Annette Johnson, Urs von Gunten, B. Wehrli, Science 2006, 313, 10721077.

[3] K. E. Murray, S. M. Thomas, A. A. Bodour, Environ. Pullut. 2010, 158, 3462-3471. 
WILEY-VCH

\section{MINIREVIEW}

[4] S. D. Richardson, S. Y. Kimura, Anal. Chem. 2016, 88, 546-582.

[5] S. D. Richardson, T. A. Ternes, Anal. Chem. 2016, 90, 398-428.

[6] S. Bolisetty, M. Peydayesh, R. Mezzenga, Chem. Soc. Rev. 2019, 48, 463-487.

[7] G. S. Miguel, S. D. Lambert, N. J. D. Graham, Wat. Res. 2001, 35, 27402748.

[8] P.C. Chiang, E.E. Chang, J.S. Wu, Water Sci. Technol. 1997, 35, 279285.

[9] S. Y. Dong, B. Zheng, F. Wang, F. Huang, Acc. Chem. Res. 2014, 47, 1982-1994.

[10] P. F. Wei, X. Z. Yan, F. H. Huang, Chem. Soc. Rev. 2015, 44, 815-832.

[11] G. C. Yu, K. H. Jie, F. H. Huang, Chem. Rev. 2015, 115, 7240-7303.

[12] X. F. Ji, M. Ahmed, L. L. Long, N. M. Khashab, F. H. Huang, J. L. Sessler, Chem. Soc. Rev. 2019, 48, 2682-2697.

[13] Y. C. Chang, Y. Jiao, H. E. Symons, J. F. Xu, C. F. J. Faul, X. Zhang, Chem. Soc. Rev. 2019, 48, 989-1003.

[14] X. J. Hu, G. Z. Xu, H. C. Zhang, M. Li, Y. Z. Tu, X. C. Xie, Y. T. Zhu, L. Jiang, X. Q. Zhu, X. W. Ji, Y. Li, A. Li, ACS Appl. Mater. Interfaces 2020 12, 12165-12175.

[15] Z. Y. Yuan, H. Q. Liu, H. X. Wu, Y. M. Wang, Q. Liu, Y. Wang, S. F. Lincoln, X. H. Guo, J. Wang, J. Chem. Eng. Data 2020, 65, 678-689.

[16] G. S. Chen, M. Jiang, Chem. Soc. Rev. 2011, 40, 2254-2266.

[17] A. Alsbaiee, B. J. Smith, L. L. Xiao, Y. H. Ling, D. E. Helbling, W. R. Dichtel, Nature 2016, 529, 190-194

[18] L. L. Xiao, Y. H. Ling, A. Alsbaiee, C. J. Li, D. E. Helbling, W. R. Dichtel, J. Am. Chem. Soc. 2017, 139, 7689-7692.

[19] M. J. Klemes, Y. H. Ling, C. Ching, C. Y. Wu, L. L. Xiao, D. E. Helbling, W. R. Dichtel, Angew. Chem. Int. Ed. 2019, 58, 12049-12053.

[20] X. M. Qin, L. Bai, Y. Z. Tan, L. Li, F. Song, Y. Z. Wang, Chem. Eng. J. 2019, 372, 1007-1018.

[21] D. S. Guo, Y. Liu, Chem. Soc. Rev. 2012, 41, 5907-5921.

[22] R. Kumar, A. Sharma, H. Singh, P. Suating, H. S. Kim, K. Sunwoo, I. Shim, B. C. Gibb, J. S. Kim, Chem. Rev. 2019, 119, 9657-9721.

[23] C. F. Chen, Y. Han, Acc. Chem. Res. 2018, 51, 2093-2106.

[24] D. Shetty, I. Jahovic, J. Raya, F. Ravaux, M. Jouiad, J. C. Olsen, A. Trabolsi, J. Mater. Chem. A. 2017, 5, 62-66.

[25] D. Shetty, J. Raya, D. S. Han, Z. Asfari, J. C. Olsen, A. Trabolsi, Chem. Mater. 2017, 29, 8968-8972.

[26] D. Shetty, I. Jahovic, J. Raya, Z. Asfari, J. C. Olsen, A. Trabolsi, ACS Appl. Mater. Interfaces 2018, 10, 2976-2981.

[27] H. C. Zhang, Z. N. Liu, Y. L. Zhao, Chem. Soc. Rev. 2018, 47, 54915528.

[28] J. Murray, K. Kim, T. Ogoshi, W. Yao, B. C. Gibb, Chem. Soc. Rev. 2017, 46, 2479-2496.

[29] K. C. Jie, Y. J. Zhou, E. R. Li, F. H. Huang, Acc. Chem. Res. 2018, 51, 2064-2072.

[30] B. B. Shi, H. X. Guan, L. Q. Shangguan, H. Wang, D. Y. Xia, X. Q. Kong, F. H. Huang, J. Mater. Chem. A. 2017, 5, 24217-24222.

[31] S. Lan, S. J. Zhan, J. M. Ding, J. Q. Ma, D. Ma, J. Mater. Chem. A. 2017 5, 2514-2518.

[32] Q. Zhao, Y. Liu, Chem. Commun. 2018, 54, 7362-7365.

[33] H. Kumari, J. Zhang, L. Erra, L. J. Barbour, C. A. Deakyne J. L. Atwood, CrystEngComm 2013, 15, 4045-4048.

[34] K. Kobayashi, M. Yamanaka, Chem. Soc. Rev. 2015, 44, 449-466.

[35] C. M. Kane, A. Banisafar, T. P. Dougherty, L. J. Barbour, K. T. Holman, J. Am. Chem. Soc. 2016, 138, 4377-4392.

[36] K. Z. Su, W. J. Wang, B. B Li, D. Q. Yuan, ACS Sustainable Chem. Eng. 2018, 6, 17402-17409.

[37] L. P. Skala, A. N. Yang, M. J. Klemes, L. L. Xiao, W. R. Dichtel, J. Am. Chem. Soc. 2019, 141, 13315-13319.

[38] H. Y. Gong, B. M. Rambo, E. Karnas, V. M. Lynch, J. L. Sessler, Nature. Chem. 2010, 2, 406-409.

[39] B. M. Rambo, H.-Y. Gong, M. Oh, J. L. Sessler, Acc. Chem. Res. 2012, 45, 1390-1401.

[40] X. F. Ji, X. D. Chi, M. Ahmed, L. L. Long, J. L. Sessler, Acc. Chem. Res. 2019, 52, 1915-1927.

[41] X. F. Ji, R. T. Wu, L. L. Long, C. X. Guo, N. M. Khashab, F. H. Huang, J. L. Sessler, J. Am. Chem. Soc. 2018, 140, 2777-2780.
[42] Z. C. Liu, S. K. M. Nalluri, J. F. Stoddart, Chem. Soc. Rev. 2017, 46, 2459-2478.

[43] H. R. Wessels, C. Slebodnick, H. W. Gibson, J. Am. Chem. Soc. 2018, 140, 7358-7370.

[44] T. L. Price Jr., H.W. Gibson, J. Am. Chem. Soc. 2018, 140, 4455-4465.

[45] D. Shetty, J. K. Khedkar, K. M. Parkad, K. Kim, Chem. Soc. Rev. 2015, 44, 8747-8761.

[46] J. D. Barrio, J. Liu, R. A. Brady, C. S. Y. Tan, S. Chiodini, M. Ricci, R. F. Leiro, C. J. Tsai, P. Vasileiadi, L. D. Michele, D. Lairez, C. Toprakcioglu, O. A. Scherman, J. Am. Chem. Soc. 2019, 141, 14021-14025.

[47] J. Liu, C. S. Y. Tan, O. A. Scherman, Angew. Chem. Int. Ed. 2018, 57, 8854-8858.

[48] D. S. Kim, J. L. Sessler, Chem. Soc. Rev. 2015, 44, 532-546.

[49] Q. He, G. I. Vargas-Zúñiga, S. H. Kim, S. K. Kim, J. L. Sessler, Chem. Rev. 2019, 119, 9753-9835.

[50] W. Chen, C. X Guo, Q. He, X. D. Chi, V. M. Lynch, Z. Y. Zhang, J. H. Su, H. Tian, J. L. Sessler, J. Am. Chem. Soc. 2019, 141, 14798-14806.

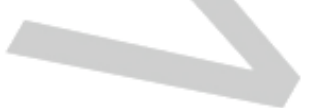




\section{WILEY-VCH}

\section{MINIREVIEW}

\section{Entry for the Table of Contents}

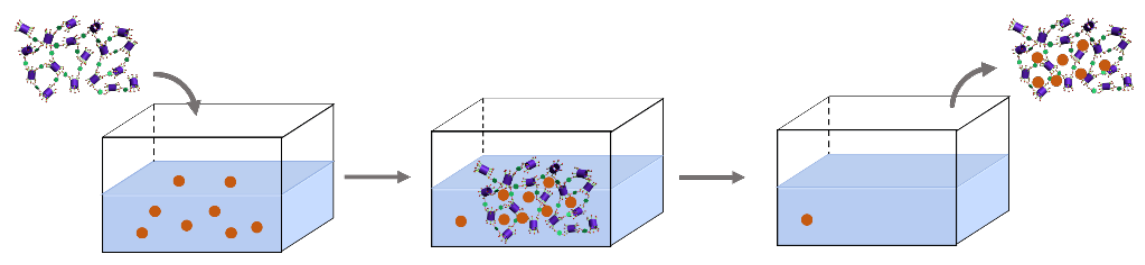

In this review, we summarize recent advances (from 2016 to 2020) involving the use of macrocycle-containing covalent polymer networks to remove organic micropollutants from water. An overview of future challenges and a perspective on this promising subfield are also provided. 